\title{
Intimate Partner Violence and Current Mental Health Needs Among Female Veterans
}

\author{
Katherine M. Iverson, PhD, Dawne Vogt, PhD, Melissa E. Dichter, PhD, \\ S. Louisa Carpenter, BS, Rachel Kimerling, PhD, Amy E. Street, PhD, and \\ Megan R. Gerber, $M D$
}

Background: The Veterans Health Administration (VHA) recommends screening female patients for intimate partner violence (IPV), yet few studies inform IPV screening efforts among this population. This study examined the proportion of women who experienced IPV within the past year and the associations between IPV and depression, post-traumatic stress disorder (PTSD), alcohol dependence, mental health multimorbidity (ie, 2 or 3 of these conditions), and military sexual trauma (MST) among female veterans.

Methods: A cross-sectional mail survey of 160 female VHA patients with an intimate partner within the past year was conducted in 2012 in New England. Self-reported IPV was assessed using the Hurt, Insult, Threaten, Scream screening tool. The survey also included validated screening measures of depression (Center for Epidemiologic Studies Depression Scale), PTSD (PTSD Checklist-Civilian), alcohol misuse (10-item Alcohol Use Disorders Identification Test), and MST.

Results: Approximately $37 \%$ of women reported IPV within the past year on the Hurt, Insult, Threaten, Scream tool. Odds ratios for the associations between reporting IPV and mental health outcomes ranged between 2.75 and 3.67. With the exception of alcohol dependence, IPV remained strongly associated with mental health conditions when adjusting for MST.

Conclusions: These findings can increase provider knowledge of the strong connection between pastyear IPV and mental health conditions among female veterans. This may encourage IPV screening and facilitate appropriate referrals, treatment conceptualization, and planning within the VHA and other health care settings. (J Am Board Fam Med 2015;28:772-776.)

Keywords: Comorbidity, Domestic Violence, Mental Health, Patient Care Team, Screening

Intimate partner violence (IPV) is a substantial threat to women's health and safety. ${ }^{1}$ Women who experience IPV may be at risk for numerous mental health conditions, ${ }^{2}$ including multimorbid mental health conditions, ${ }^{3}$ which can make it difficult to

This article was externally peer reviewed.

Submitted 7 May 2015; revised 29 June 2015; accepted 7 July 2015.

From the National Center for PTSD, VA Boston Healthcare System, Boston, MA (KMI, DV, SLC, AES); the Department of Psychiatry, Boston University School of Medicine, Boston, MA (KMI, DV, AES); the Center for Health Equity Research and Promotion, Philadelphia VA Medical Center, Philadelphia, PA (MED); the National Center for PTSD, VA Palo Alto Health Care System, Menlo Park, CA (RK); the Center for Innovation to Implementation, VA Palo Alto Health Care System, Menlo Park, CA (RK); the Women's Health Center, VA Boston Healthcare System, Boston, MA (MRG); and the Department of Medicine, Boston University School of Medicine, Boston, MA (MRG).

Funding: This work was supported by the Department of Veterans Affairs, Veterans Health Administration, Health break free of violence and recover from IPV. However, adequate treatment of mental health conditions reduces women's risk for subsequent IPV. ${ }^{4}$ Primary care providers (PCPs) are in an ideal position to identify and refer to mental health care female patients who experience IPV. Research is needed to help physicians increase identification

Services Research and Development (HSR\&D) Services as part of HSR\&D Career Development Awards (CDA 10-029 to KMI, and CDA 10-202 to MED) and a Presidential Early Career Award for Scientists and Engineers (USA 14-275 to $\mathrm{KMI})$.

Conflict of interest: none declared.

Disclaimer: The views expressed in this article are those of the authors and do not necessarily represent the views of the Department of Veterans Affairs or the United States Government.

Corresponding author: Katherine M. Iverson, $\mathrm{PhD}, \mathrm{VA}$ Boston Healthcare System, 150 S. Huntington Ave., 116B-3, Boston, MA 02130 (E-mail: katherine.iverson@va. gov). 
and appropriate mental health referrals, as well as facilitate multidisciplinary treatment planning.

Although there is relatively little work examining IPV and health correlates among military veteran women relative to civilian women, female veterans may be at heightened risk for IPV. ${ }^{5}$ The Veterans Health Administration (VHA) recommends that providers screen female patients for IPV within the past year. ${ }^{6}$ Although VHA providers conduct mandated screening for depression, post-traumatic stress disorder (PTSD), alcohol use disorder, and military sexual trauma (MST; repeated, threatening sexual harassment or sexual assault during military service), IPV screening remains a recommendation rather than a requirement. ${ }^{6} \mathrm{VHA}$ providers are favorably disposed to IPV screening; however, a lack of knowledge about IPV prevalence and its connection to mental health remain barriers to screening. ${ }^{7}$ Research is needed to inform PCPs' expectations regarding the rates at which female veterans screen positive for past-year IPV as well as to increase their understanding of the strength of the associations between IPV and prevalent mental health disorders among this population. This type of knowledge may encourage the adoption of IPV screening practices and aid in treatment planning.

The aims of this survey study were to (1) identify the proportion of women who screen positive for past-year IPV among a sample of female veteran VHA patients, and (2) examine the strength of the associations between IPV and depression, PTSD, alcohol dependence, and mental health multimorbidity while adjusting for demographics and MST. We hypothesized that past-year IPV would be associated with a higher risk for experiencing these mental health conditions. Because MST is associated with increased risk for mental health disorders $^{8}$ and IPV, ${ }^{9}$ we also examined the association of IPV with mental health symptoms, adjusting for MST history.

\section{Methods \\ Participants}

Participants were 160 female veterans participating in a larger study. ${ }^{10}$ Using the VHA Corporate Data Warehouse, 700 women were randomly selected from the population of female veteran VHA patients in New England: 581 with locatable addresses received a survey, 21 declined participation, 191 did not return a survey, and 369 responded
(63.5\% response rate). Comparisons between responders and nonresponders on demographics from administrative records revealed a difference in age: responders were slightly older (Cohen $\mathrm{d}=$ 0.27). Participants in this substudy were the 160 women who reported being involved in an intimate relationship in the past year.

\section{Procedure}

As described elsewhere, ${ }^{10}$ surveys were mailed to potential participants using a modified multiple mailing strategy ${ }^{11}$ : (1) informed consent fact sheet, survey packet, and \$10 incentive; (2) 2 weeks later a thank you/reminder postcard; and (3) 4 weeks later an additional survey packet and $\$ 10$ incentive to nonresponders. The local institutional review board approved all procedures.

\section{Measures}

\section{IPV Screen}

The Hurt, Insult, Threaten, Scream (HITS) tool ${ }^{12}$ comprises 4 items: "Over the past 12 months, how often did your partner: (1) physically hurt you?, (2) insult you or talk down to you?, (3) threaten you with harm?, and (4) scream or curse at you?" Responses are scored on a 5-point scale from 1 ("never") to 5 ("frequently") and summed for a total score. Consistent with prior work demonstrating that a cutoff score of 6 optimizes the sensitivity and specificity of the HITS in detecting IPV among female VHA patients, ${ }^{10,13}$ women who scored $\geq 6$ screened positive for past-year IPV.

\section{Mental Health}

Depression was measured using the 20 -item Center for Epidemiologic Studies Depression Scale. ${ }^{14} \mathrm{~A}$ cutoff score of 16 was used to develop a dichotomous probable depression variable. ${ }^{14}$ PTSD was measured using the 17-item PTSD Checklist. ${ }^{15} \mathrm{~A}$ cutoff score of 50 was used to develop a dichotomous probable PTSD variable. ${ }^{15}$ Alcohol dependence was measured using the 10-item Alcohol Use Disorders Identification Test. ${ }^{16}$ A cutoff score of 8 was used to develop a dichotomous probable alcohol dependence variable. ${ }^{16}$ Mental health multimorbidity was defined as the presence of at least 2 of these self-reported conditions. ${ }^{17}$

\section{MST}

Two items from the Trauma Questionnaire ${ }^{18}$ assessed MST: "While you were in the military, did 
Table 1. Unadjusted Associations Between Reports of Past-Year Intimate Partner Violence Experiences and Mental Health Conditions Among a 2012 Mail Survey Sample of Female Veterans Health Administration Patients

\begin{tabular}{lccc}
\hline $\begin{array}{l}\text { Probable Mental } \\
\text { Health Condition }\end{array}$ & $\begin{array}{c}\text { Odds } \\
\text { Ratio }\end{array}$ & $\begin{array}{c}\text { 95\% Confidence } \\
\text { Interval }\end{array}$ & $\begin{array}{c}P \\
\text { Value }\end{array}$ \\
\hline Model 1: depression & 3.21 & $1.63-6.34$ & .001 \\
Model 2: PTSD & 2.75 & $1.34-5.64$ & .006 \\
$\begin{array}{l}\text { Model 3: alcohol } \\
\text { dependence }\end{array}$ & 3.06 & $1.05-8.93$ & .040 \\
$\begin{array}{c}\text { Model 4: } \geq 2 \mathrm{MH} \\
\text { conditions* }\end{array}$ & 3.67 & $1.81-7.43$ & .001 \\
\hline
\end{tabular}

Because of missing data, the number of patients varied from 152 to 158. Cases were excluded via list-wise deletion because of missing values.

*Mental health (MH) multimorbidity, defined as the presence of 2 or 3 of the $\mathrm{MH}$ conditions examined in this study.

PTSD, post-traumatic stress disorder.

you receive uninvited and unwanted sexual attention, such as touching or cornering, pressure for sexual favors, or verbal remarks?" and "While you were in the military, did someone ever use force or the threat of force to have sexual contact with you?" A positive response to either item indicated a history of MST.

\section{Demographics}

Self-report questions developed for this study assessed age, race/ethnicity, education, marital status, service branch, and rank.

\section{Data Analysis}

We examined crude associations between past-year IPV (IPV positive/IPV negative) and the 3 mental health conditions (yes/no), as well as mental health multimorbity (yes/no), as outcome variables in 4 separate logistic regression models. Next, we conducted logistic regression analyses for the 4 outcome variables while controlling for MST. We also controlled for age and race because these factors have been associated with mental health outcomes among female veterans. ${ }^{6,19}$ We calculated 95\% confidence intervals for odds ratios and adjusted odds ratios. Analyses were performed with SPSS (IBM Corp., Armonk, NY).

\section{Results}

The average age of participants was 47.7 years (standard deviation, 14.2 years). Of the women,
$80 \%$ identified as white, $57.5 \%$ were currently married, and $60.4 \%$ had less than a college degree. Half the sample served in the Army and most (89\%) were enlisted (vs officers). A total of 59 participants $(36.9 \%)$ screened positive for past-year IPV. There were no significant differences in sociodemographic variables among women who did and did not screen positive for IPV on the HITS.

Table 1 displays the unadjusted associations between IPV and mental health outcomes. Women who reported IPV were 3.21, 2.75, and 3.06 times more likely to experience depression, PTSD, and alcohol dependence, respectively, than women who did not report IPV. Similarly, women who reported IPV were 3.67 times more likely to experience mental health multimorbidity.

Table 2. Associations Between Reports of Past-Year Intimate Partner Violence Experiences and Mental Health Conditions, Adjusted for Military Sexual Trauma

\begin{tabular}{lccc}
\hline $\begin{array}{l}\text { Probable Mental Health } \\
\text { Condition }\end{array}$ & $\begin{array}{c}\text { Adjusted } \\
\text { OR }\end{array}$ & $\begin{array}{c}\text { Confidence } \\
\text { Interval }\end{array}$ & $\begin{array}{c}P \\
\text { Value }\end{array}$ \\
\hline $\begin{array}{l}\text { Model 1: depression } \\
\text { Age }\end{array}$ & 0.93 & $0.64-1.34$ & .69 \\
Race (white vs nonwhite) & 0.39 & $0.16-0.94$ & .04 \\
MST & 2.33 & $1.16-4.69$ & .02 \\
IPV & 3.02 & $1.46-6.26$ & .003 \\
Model 2: PTSD & & & \\
Age & 1.18 & $0.79-1.77$ & .42 \\
Race (white vs. nonwhite) & 0.36 & $0.15-0.87$ & .02 \\
MST & 2.98 & $1.29-6.84$ & .01 \\
IPV & 2.35 & $1.08-5.08$ & .031 \\
Model 3: alcohol dependence & & & \\
Age & 0.86 & $0.50-1.49$ & .59 \\
Race (white vs. nonwhite) & 0.51 & $0.16-1.67$ & .27 \\
MST & 1.38 & $0.43-4.45$ & .59 \\
IPV & 2.88 & $0.94-8.82$ & .06 \\
Model 4: $\geq 2$ MH & & & \\
$\quad$ conditions* & & & .002 \\
Age & 1.20 & $0.80-1.80$ & .37 \\
Race (white vs. nonwhite) & 0.30 & $0.12-0.75$ & .01 \\
MST & 2.83 & $1.27-6.30$ & .01 \\
IPV & 3.32 & $1.54-7.17$ & .002 \\
\hline & & & \\
\hline
\end{tabular}

Because of missing data, the numbers of patients varied from 152 to 158 . Cases were excluded via list-wise deletion because of missing values.

* Mental health (MH) multimorbidity, defined as the presence of 2 or 3 of the $\mathrm{MH}$ conditions examined in this study.

IPV, intimate partner violence; MST, military sexual trauma; OR, odds ratio; PTSD, post-traumatic stress disorder. 
Even when accounting for MST, age, and race, women reporting IPV were 3.02, 2.35, and 3.32 times more likely to meet criteria for depression, PTSD, and mental health multimorbidity, respectively (Table 2). However, neither IPV nor MST remained significantly associated with alcohol dependence when examined simultaneously in the model. In addition, with the exception of alcohol dependence, race was associated with mental health outcomes such that identifying as white was associated with lower risk for mental health outcomes. Age was not significantly associated with mental health outcomes.

\section{Discussion}

Findings demonstrate markedly increased odds of self-reported depression, PTSD, and alcohol dependence, as well as mental health multimorbidity, among the substantial proportion of female veterans who experienced past-year IPV in this study. Results remained robust for depression, PTSD, and mental health multimorbidity when adjusting for MST, whereas a substantial, but nonsignificant, effect remained for alcohol dependence. Results provide an important extension of prior work ${ }^{1,20}$ by controlling for MST, a common stressor experienced by female veterans that negatively affects mental health. ${ }^{8}$ This study also documents the connection between IPV and common mental health conditions in an understudied population-female VHA patients-while accounting for race and age, factors that are associated with mental health outcomes among this population. ${ }^{19}$

The importance of screening female veteran patients for IPV and providing appropriate counseling interventions is underscored by the high proportion of women $(37 \%)$ in this sample who screened positive for IPV. This finding is consistent with another study of partnered female veterans ${ }^{13}$ and in the upper range of what has been found among female health care patients. ${ }^{21}$ Yet research with both patients and providers indicate that health services often fail to identify and address IPV with patients, or neglect the role of IPV in contributing to mental health conditions. ${ }^{22,23}$ The robust associations between past-year IPV and current mental health conditions may promote the uptake of IPV screening by VHA and other health care providers by increasing education about IPV and its mental health effects, a factor that providers indicated would facilitate the adoption of IPV screening practices. ${ }^{7}$ PTSD, depression, and alcohol dependence are routinely identified and treated in primary care and mental health settings, and an annual clinical reminder for an IPV screening tool such as the HITS could be embedded within the electronic medical record. Being aware that a patient has recently experienced IPV, while understanding the connection between IPV and mental health, will allow providers to better coordinate care and individually tailor treatment.

This cross-sectional study used self-report screening measures of IPV and mental health conditions. Although these measures are reliable and valid, replicating these findings in clinical settings using comprehensive assessments of IPV and mental health, including conditions not examined here, remains important. Nonresponse bias may have affected the rate of IPV found in this study because younger women were less likely to participate. In addition, findings may not generalize to all female veterans.

\section{Conclusion}

These findings underscore the importance of PCPs' willingness and ability to detect IPV and address mental health conditions (eg, screening, brief intervention, and referral), as well as the importance of ensuring the availability of mental health providers for the delivery of comprehensive care. With adequate training and support, PCPs can play a critical role in IPV identification and facilitating mental health treatment in this population. Although there continues to be exponential growth in the number of female veterans seeking care from the VHA, ${ }^{19}$ many women seek care outside the $\mathrm{VHA},{ }^{24}$ underscoring the relevance of these findings for both VHA and non-VHA providers.

\section{References}

1. Gerber MR, Iverson KM, Dichter ME, et al. Women veterans and intimate partner violence: current state of knowledge and future directions. J Womens Health 2014;23:302-9.

2. Trevillion K, Oram S, Feder G, et al. Experiences of domestic violence and mental disorders: a systematic review and meta-analysis. PloS One 2012; $7: e 51740$.

3. Cerulli C, Talbot NL, Tang W, et al. Co-occurring intimate partner violence and mental health diagno- 
ses in perinatal women. J Womens Health 2011;20: 1797-803.

4. Iverson KM, Gradus JL, Resick PA, et al. Cognitivebehavioral therapy for PTSD and depression symptoms reduces risk for future intimate partner violence among interpersonal trauma survivors. J Consult Clin Psychol 2011;79:193-202.

5. Dichter ME, Cerulli C, Bossarte RM. Intimate partner violence victimization among women veterans and associated heart health risks. Womens Health Issues 2011;21(4 Suppl):S190-4.

6. Veterans Health Administration Domestic Violence Task Force. Plan for implementation of the Domestic Violence/Intimate Partner Violence Assistance Program. Washington, DC: Department of Veterans Affairs, Veterans Health Administration; 2013.

7. Iverson KM, Wells ST, Wiltsey-Stirman S, et al. VHA primary care providers' perspectives on screening female veterans for intimate partner violence: a preliminary assessment. J Fam Violence 2013;28: 823-31.

8. Kimerling R, Street AE, Pavao J, et al. Militaryrelated sexual trauma among Veterans Health Administration patients returning from Afghanistan and Iraq. Am J Public Health 2010;100:1409-12.

9. Iverson KM, Mercado R, Carpenter SL, Street AE. Intimate partner violence among women veterans: previous interpersonal violence as a risk factor. J Trauma Stress 2013;26:767-71.

10. Iverson KM, King M, Resick PA, et al. Clinical utility of an intimate partner violence screening tool for female VHA patients. J Gen Intern Med 2013; 28:1288-93.

11. Dillman DA. Mail and internet surveys: the tailored design method. Hoboken, NJ: John Wiley and Sons; 2007.

12. Sherin KM, Sinacore JM, Li XQ, et al. HITS: a short domestic violence screening tool for use in a family practice setting. Fam Med 1998;30:508-12.

13. Iverson KM, King MW, Gerber MR, et al. Accuracy of an intimate partner violence screening tool for female VHA patients: a replication and extension. J Trauma Stress 2015;28:79-82.

14. Radloff LS. The CES-D Scale: a self-report depression scale for research in the general population. Appl Psychol Meas 1977;1:385-401.
15. Weathers F, Litz BT, Herman DS, et al. The PTSD Checklist (PCL): reliability, validity, and diagnostic utility. Presentation at the International Society for Traumatic Stress Studies; San Antonio, TX; 1993.

16. Babor TF, Higgins-Biddle JC, Saunders JB, et al. The Alcohol Use Disorders Identification test: guidelines for use in primary care. Geneva, Switzerland: World Health Organozation, Department of Mental Health and Substance Dependence; 2001.

17. Batstra L, Neelemanb J, Elsingac C, Hadders-Algrad M. Psychiatric morbidity is related to a chain of prenatal and perinatal adversities. Early Hum Dev 2006;82:721-9.

18. McIntyre LM, Butterfield MI, Nanda K, et al. Validation of a trauma questionnaire in veteran women. J Gen Intern Med 1999;14:186-9.

19. Frayne SM PC, Saechao F, Maisel NC, et al. Sourcebook: women veterans in the Veterans Health Administration. Vol. 3: Sociodemographics, utilization, costs of care, and health profile. Washington D.C.: Women's Health Evaluation Initiative, Veterans Health Administration, Department of Veterans Affairs; 2014.

20. Dichter, Marcus SC, Wagner C, Bonomi AE. Associations between psychological, physical, and sexual intimate partner violence and health outcomes among women veteran VA patients. Soc Work Ment Health 2014;12:411-28.

21. Nelson HD, Bougatsos C, Blazina I. Screening women for intimate partner violence: a systematic review to update the U.S. Preventive Services Task Force recommendation. Ann Intern Med 2012;156: 796-808.

22. Trevillion K, Howard LM, Morgan C, et al. The response of mental health services to domestic violence: a qualitative study of service users' and professionals' experiences. J Am Psychiatr Nurses Assoc 2012;18:326-36.

23. Trevillion K, Hughes B, Feder G, et al. Disclosure of domestic violence in mental health settings: a qualitative meta-synthesis. Int Rev Psychiatry 2014;26: 430-44.

24. Washington DL, Bean-Mayberry B, Hamilton AB, Cordasco KM, Yano EM. Women veterans' healthcare delivery preferences and use by military service era: findings from the National Survey of Women Veterans. J Gen Intern Med 2013;28:S571-6. 